\title{
Computer-assisted anti-AIDS drug development: cyclophilin B against the HIV-1 subtype A V3 loop
}

\author{
Alexander M. Andrianov ${ }^{1 *}$, Ivan V. Anishchenko ${ }^{2}$ \\ ${ }^{1}$ Institute of Bioorganic Chemistry, National Academy of Sciences of Belarus, Minsk, Belarus; \\ *Corresponding Author: andrianov@iboch.bas-net.by \\ ${ }^{2}$ United Institute of Informatics Problems, National Academy of Sciences of Belarus, Minsk, Belarus
}

Received 16 December 2009; revised 24 March 2010; accepted 26 March 2010.

\section{ABSTRACT}

Aim: The objects of this study originated from the experimental observations, whereby the HIV -1 gp120 V3 loop is a high-affinity ligand for immunophilins, and consisted in generating the structural complex of cyclophilin (Суc) B belonging to immunophilins family with the virus subtype A V3 loop (SA-V3 loop) as well as in specifying the Cyc $B$ segment forming the binding site for V3 synthetic copy of which, on the assumption of keeping the 3D peptide structure in the free state, may present a forwardlooking basic structure for anti-AIDS drug development. Methods: To reach the objects of view, molecular docking of the HIV-1 SA-V3 loop structure determined previously with the X-ray conformation of Cyc B was put into practice by Hex 4.5 program (http://www.loria.fr/ ritchied/ hexl) and the immunophilin stretch responsible for binding to V3 (Cyc B peptide) was identified followed by examination of its 3D structure and dynamic behavior in the unbound status. To design the Cyc B peptide, the X-ray conformation for the identical site of the native protein was involved in the calculations as a starting model to find its best energy structural variant. The search for this most preferable structure was carried out by consecutive use of the molecular mechanics and simulated annealing methods. The molecular dynamics computations were implemented for the Сyc B peptide by the GROMACS computer package (http:Il www.gromacs.orgl). Results: The overmolecular structure of Cyc B with V3 was built by computer modeling tools and the immunophilinderived peptide able to mask effectively the structurally invariant V3 segments embracing the functionally crucial amino acids of the HIV-1 gp120 envelope protein was constructed and analyzed. Conclusions: Starting from the joint analysis of the results derived with those of the literature, the generated peptide was suggested to offer a promising basic structure for making a reality of the protein engineering projects aimed at developing the anti-AIDS drugs able to stop the HIV's spread.

Keywords: HIV-1; V3 Loop; Cyclophilin B; Computer Modeling; Molecular Docking;

Anti-Aids Drug Design

\section{INTRODUCTION}

The HIV-1 envelope glycoprotein (Env), the etiologic agent of AIDS [1], consists of two noncovalently bound subunits derived from the gp160 precursor. One of these subunits, gp120 protein, is localized on the surface of the viral isolates and becomes a direct party to the virus binding to the target-cells, whereas the other, transmembrane gp41 protein, triggers the process of membrane fusion resulting in the invasion of the virus genome into the macrophages and T-lymphocytes [2]. Specific interactions of the HIV-1 with the virus primary receptor CD4 as well as with its chemokine co-receptors CCR5 and/or CXCR4 are put into effect using the V1-V5 loops of gp120 disclosing the high variability of the amino acid sequences in diverse virus strains [3-5]. Currently, special emphasis of the research teams involved in the anti-AIDS drug studies is attracted to the HIV-1 V3 loop (reviewed in [6]). The higher interest in V3 is caused by numerous experimental data [7] testifying to the fact that exactly this gp120 site gives rise to the principal target for neutralizing antibodies and accounts for the choice of co-receptor determining the preference of the virus in respect with T-lymphocytes or primary macrophages [8]. The differential usage of co- 
receptors, which is critically dependent on the sequence, charge, and/or structure of the V3 region of gp120 $[9,10]$, dictates the viral phenotype, which shows a typical pattern of evolution during the natural history of HIV-1 infection. CCR5-restricted strains (R5) are the most prevalent in vivo, as they are almost invariably responseble for the initial transmission, predominate during the long asymptomatic phase of the infection, and often persist after the progression to full-blown AIDS; by contrast, strains that utilize CXCR4, either alone (X4) or in combination with CCR5 (R5X4), emerge only in a subset of patients, typically in conjunction with the onset of clinical signs of disease progression and immune system deterioration $[11,12]$.

Since the V3 loop governs the cell tropism and cell fusion [7], one of the strategic ways in developing the anti-HIV-1 drugs may be based on the approach anticipating the search for the chemicals able to block efficiently this functionally significant stretch of gp120 [6]. Comprehensive analysis of the data given in study [13] allows one to suppose that immunophilins exhibiting specific high-affinity interactions with the HIV-1 V3 loop may be utilized as a basic substance to set out of the search for the potential anti-AIDS therapeutic agents.

Immunophilins known originally as the cellular receptors of the immunosuppressive drugs cyclosporine A and FK506 or rapamycin organize the extensive group of proteins exhibiting peptidyl-prolyl cis-trans isomerase activity which is inhibited specifically and efficiently on binding of the corresponding immunosuppressant [14]. Immunophilins subdivided into three families of proteins, namely cyclophilins and FK506-binding proteins (FK $\mathrm{BP}_{\mathrm{S}}$ ), and a novel chimeric dual-family immunophilin, named FK506- and Cyclosporine-binding protein (FCBP) show similar enzymatic and biological functions despite the apparent difference in their sequence and three-dimensional structures [15]. Alongside with the function of intracellular receptors of immunosuppressants, individual representatives of immunophilins act as catalysts of protein folding and as shaperones stabilizing proteins in a defined conformation and supervising the quality of their spatial structure [16,17]. A variety of bacterial and protozoan pathogens express FKBP-related peptidyl- prolyl cis-trans isomerases termed macrophage-infectivity potentiators (Mip). Mip proteins act in host cell infection as virulence factors, either as membrane-bound proteins on the surface of the pathogens or as soluble secreted proteins $[18,19]$. The peptidyl-prolyl cys-trans isomerase activity of Mip proteins is suppressed by FK506, which reduces the infectivity of the pathogens without affecting the rate of intracellular replication. Distinct immunophilins were found to be released from cells. Cyclophilin B was detected in human milk [20] and blood plasma [21], but is mainly localized in the endoplasmatic reticulum of cells. The cytosolic immunophilins cyclophin A and FKBP12 were shown to be released during apoptosis of fibroblasts [22] and to act as chemokines by unknown mechanism [23-25]. Recent researches have revealed that many immunophilins possess a shaperone function independent of peptidyl-prolyl cis-trans isomerase activity (reviewed in [15]). Knockout animal studies have confirmed multiple essential roles of immunophilins in physiology and development consisting in interactions with proteins to guide their proper folding and assembly [15].

Reasoning from the empirical observations, there is a good motive to think that immunophilins present in normal human blood plasma are directly relevant to the HIV-1 replication assisting the virus with getting into macrophages and T-lymphocytes [26]. In particular, cyclophilin A packaged into nascent virus particles by specific binding to the capsid region of the Gag precursor protein at the time of viral assembly [27-29], was found to mediate the HIV-1 attachment to the target cells via heparans followed by the gp120-CD4 interaction [26]. Due to the interaction of immunophilins with the HIV-1 isolates, their role of conformases or docking mediators in the virus life cycle seems to be highly probable, since immunophilin receptors on cell membranes and immunophilin-related virulence factors of pathogens have been identified [13].

This work proceeds with our previous studies [30,31] where two virtual molecules, namely FKBP and Cyc A peptides, presenting the promising anti-HIV-1 pharmacological substances were designed by means of computer modeling based on the analysis of specific interactions of the FK506-binding protein and cyclophilin A with V3.

The object of the present study was to model the structural complex of one more protein from immunophilins superfamily, cyclophilin (Cyc) B, with the HIV-1 subtype A V3 loop (SA-V3 loop) circulating in Eastern Europe including Republic of Belarus and, therefore, offering the target of our special interest, as well as to specify the Cyc B segment forming the binding site for V3, the synthetic copy of which, on the assumption of keeping the 3D peptide structure in the free state, may be considered as a forward-looking applicant for the role of a new antiviral drug.

To this effect, molecular docking of the HIV-1 SA-V3 structure determined previously [32] with the X-ray conformation of $\mathrm{Cyc} \mathrm{B}$ was put into practice, and the Cyc B stretch responsible for the binding to V3 was identified followed by predicting the most probable 3D structure of this stretch in the unbound state, studying its dynamic behavior, and collating the results obtained 
with the X-ray data for the corresponding site of Cyc B. Thereupon, the potential energy function was analyzed for the complex of the SA-V3 loop with the Cyc B peptide offering the virtual molecule that imitates the $\mathrm{Cyc} B$ segment making a key contribution to the interactions of the native protein with V3. As a matter of record, the designed peptide was shown to be capable of the effecttive masking of the functionally critical and structurally rigid V3 sites, presenting the suitable framework for making a reality of the protein engineering projects utilizing the V3 target for developing the anti-AIDS drugs able to stop the HIV's spread.

\section{METHODS}

\subsection{Molecular Docking Simulations}

Molecular docking of the SA-V3 loop [32] with Cyc B (file 1CYN of the Protein Data Bank [33,34]) as well as with the Cyc B peptide was executed by the Hex 4.5 program [35] which presents an interactive molecular graphics package for calculating and displaying feasible docking modes of pairs of protein and DNA molecules and employs the spherical polar Fourier correlations to accelerate the computations. Energy refinement of the generated complexes was performed in the GROMACS package [36] by minimizing their potential energy. To this end, the conjugate gradient method was used for the complex of the native protein with the V3 loop as well as for the overmolecular ensemble of V3 with the Cyc B peptide. At the final point of computations, the structural complexes were subjected to the procedure of simulating annealing carried out during 100 ps time domain at initial and final temperatures equal to 500 and $0 \mathrm{~K}$ respecttively.

\subsection{Determination of the 3D Static Structure for the Cyc B Peptide and Molecular Dynamics Computations}

To design the $3 \mathrm{D}$ structure of the $\mathrm{Cyc} \mathrm{B}$ peptide, the $\mathrm{X}$-ray conformation of the Cyc $\mathrm{B}$ site [37] responsible for its binding to $\mathrm{V} 3$ was involved in the calculations as a starting model to find its best energy structural variant in the unbound form. The search for this most preferable conformation was executed by consecutive use of the molecular mechanics and simulated annealing methods realized in the Tinker package [38] with activating its program modules Minimize and Anneal.

The molecular dynamics (MD) simulations of the built Cyc B peptide structure were implemented by the GROMACS computer package [36] using the GROMOS96 force field parameter set 53A6 [39]. The starting $3 \mathrm{D}$ structure of the $\mathrm{CycB}$ peptide generated hereinbefore was placed in a cubic box so that the smallest distance between its walls and the peptide atoms was greater than the half of the cut-off radius of the Coulomb and Lennard-Jones potentials fixed at $1.4 \mathrm{~nm}$. Simple point charge water model [40] was utilized to set the parameters of explicit solvent on which the periodic boundary conditions were imposed in all directions. Before the MD computations, the initial Cyc B peptide model was subjected to the procedure of energy minimization realized in vacuum by the steepest descent method. The MD simulations were carried out at temperature $310 \mathrm{~K}$ during $20.5 \mathrm{~ns}$ time domain with 1 fs step at fixed pressure and number of atoms, the first $0.5 \mathrm{~ns}$ being the stage of solvent relaxation. To integrate the Newton's equations of motion, the common leap-frog algorithm was used. To control the temperature, the weak coupling scheme to an external bath [41] was employed in the calculations with 0.1 ps characteristic time. As with the temperature coupling, the system was linked to a "pressure bath" by exponential relaxation of pressure [41] with $1.0 \mathrm{ps}$ time constant.

Every $10 \mathrm{ps,} \mathrm{the} \mathrm{geometric} \mathrm{parameters} \mathrm{of} \mathrm{the} \mathrm{MD}$ structures and the data on their energy characteristics were recorded into the trajectory file. Comparison of the MD conformations between themselves and with the input structure was performed in terms of the values of root-mean-square deviations computed both in Cartesian and angular space. To this effect, the GROMACS routines [36] were implicated in the studies.

The computations were run in parallel on SKIF K-1000 computer cluster on 64 CPUs [42].

\subsection{Identification of Secondary Structures in the Cyc B Peptide}

To determine the different types of secondary structures in the Cyc B peptide, the $\phi, \psi$ values for all of the amino acids derived from the simulated model were analyzed in compliance with the criteria given in study [43]. The types of $\beta$ - and $\gamma$-turns were identified within the classification of Hutchinson and Thornton [44]. To detect the nonstandard $\beta$-turns, the additional information on the distances $\mathrm{C}^{\alpha}{ }_{\mathrm{i}} \ldots \mathrm{C}^{\alpha}{ }_{\mathrm{i}+3}$ computed from the atomic coordinates of the simulated structures was employed.

\subsection{Collation of 3D Static Structures}

The values of root-mean-square deviations (RMSD) in atomic coordina tes (cRMSD) were taken to evaluate the similarity of the structures in the Cartesian space [45]. To compare the structures in terms of the dihedrals, the RMSD between corresponding angles (aRMSD) were used as a measure of their conformational similarity in the angular space [45]. 


\section{RESULTS AND DISCUSSION}

Figure 1 casts light on the structural complex of the HIV-1 SA-V3 loop with Cyc B generated via molecular docking of their 3D structures followed by optimization of its geometric parameters. Insight into the function describing the energy surface of the built complex makes it clear that the binding of V3 to Cyc B initiates the formation of stable overmolecular structure that is characterized by the value of potential energy equal to -6434 $\mathrm{kcal} / \mathrm{mol}$. Analysis of the matrix of interatomic contacts coming true in the designed complex allows one to identify the amino acids of V3 and Cyc B participating in the intermolecular interactions the total energy of which comes to $-75 \mathrm{kcal} / \mathrm{mol}$. So, according to the data obtained, such V3 residues as Ser-11, Val-12, Gly-15, Pro-16,
Gly-17, Gln-18, Ala-19, Thr-23, and Arg-31 take up positions nearby the surface of Cyc B giving rise to the binding site for V3 by means of Gly-1, Pro-2, Lys-3, Gly-28, Lys-29, Thr-30, Lys-91, Lys-93, and Glu-178. One needs to note that cooperation of the V3 loop with Cyc $B$ results in the origin of one ion pair organized by Arg-31 of V3 and Glu-178 of Cyc B and in the formation of six H-bonds that appear as a result of donor-acceptor interactions of the receptor amino acids Lys-91, Lys-93, and Glu-178 on the one hand as well as of V3 residues Ser-11, Val-12, Gln-18, Ala-19, and Thr-23 on the other hand (see information given in Table 1).

These results signify that interaction of the V3 loop with Cyc B entails the blockade of its central region making the immunogenic crown of gp120 [46], whereas the residues of V3 $\mathrm{N}$ - and $\mathrm{C}$-terminal segments (except

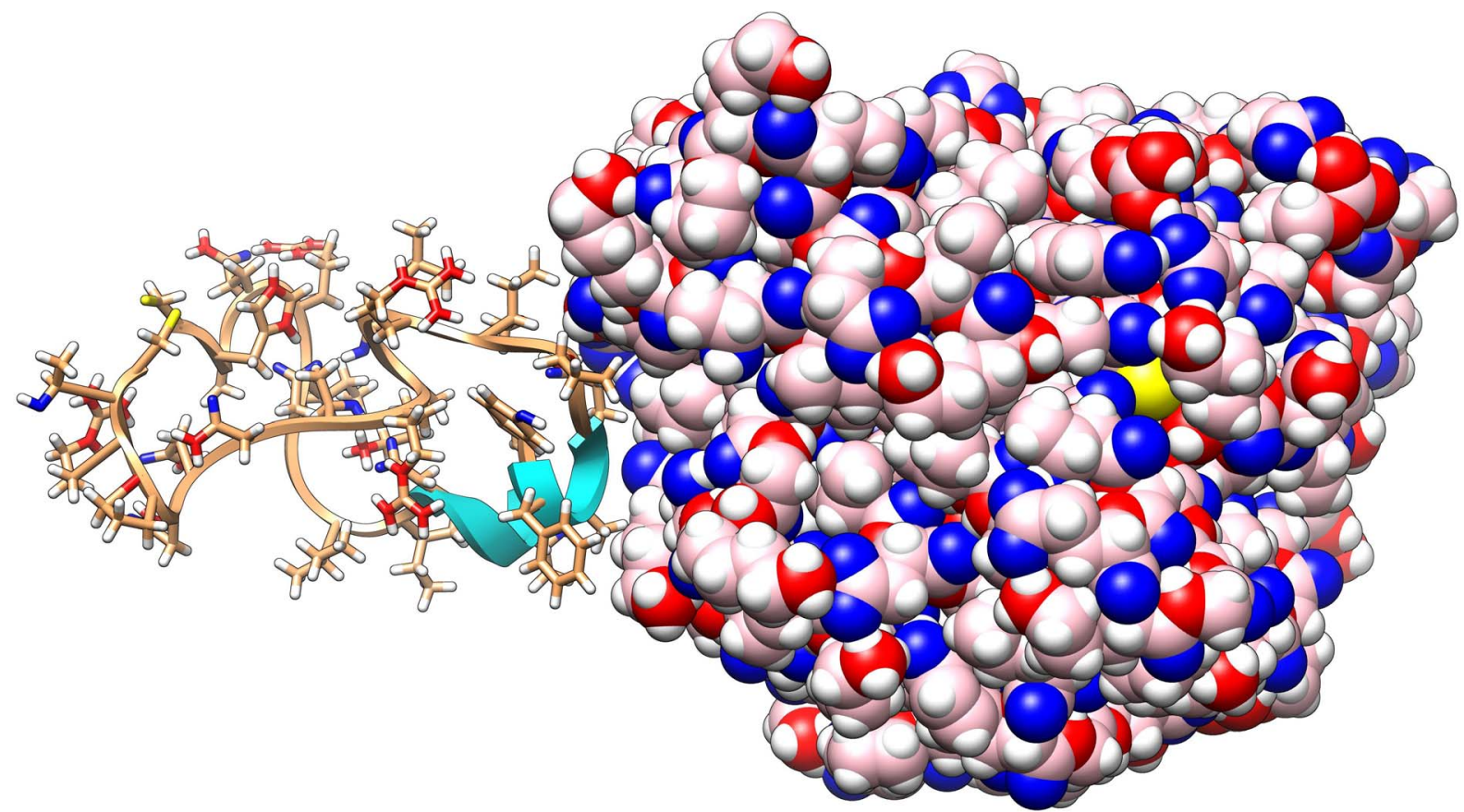

Figure 1. Image of the structural complex between the HIV-1 SA-V3 loop (tubes) and Cyc B (balls).

Table 1. Geometric parameters of intermolecular H-bonds for the structural complex of the HIV-1 SA-V3 loop with Cyc B.

\begin{tabular}{|c|c|c|c|c|c|}
\hline $\begin{array}{l}\text { Residue } \\
\text { (donor) }\end{array}$ & $\begin{array}{l}\text { Group } \\
\text { (donor) }\end{array}$ & $\begin{array}{l}\text { Residue } \\
\text { (acceptor) }\end{array}$ & $\begin{array}{l}\text { Group } \\
\text { (acceptor) }\end{array}$ & $\begin{array}{c}\text { Distance }(\AA) \\
\text { Donor...Acceptor }\end{array}$ & $\begin{array}{c}\text { Distance }(\AA) \\
\text { Hydrogen...Acceptor }\end{array}$ \\
\hline Lys- $93^{2}$ & $\mathrm{NH}$ & Gln-18 & OE1 & 2.7 & 1.7 \\
\hline Lys- $93^{2}$ & $\mathrm{NZ}$ & Thr- $23^{1}$ & OG1 & 2.8 & 1.8 \\
\hline Ser- $11^{1}$ & OG & Glu- $178^{2}$ & OE2 & 2.7 & 1.7 \\
\hline Gln-18 ${ }^{1}$ & $\mathrm{NH}$ & Lys- $91^{2}$ & $\mathrm{CO}$ & 2.8 & 1.9 \\
\hline Gln-18 ${ }^{1}$ & NE2 & Glu- $178^{2}$ & OE2 & 2.8 & 1.9 \\
\hline Ala- $19^{1}$ & $\mathrm{NH}$ & Lys- $91^{2}$ & $\mathrm{CO}$ & 3.0 & 2.0 \\
\hline
\end{tabular}

Footnote: Superscripts 1 and 2 denote the amino acids of V3 and Cyc B respectively. 
for Arg-31) relating to its stem [46] do not come in direct contact with the receptor. The data above are in harmony with those of study [13], whereby high affinity to immunophilins is typical not merely for intact V3 variable loops but also for their peptides embracing the immunogenic tip of gp120. Among the segments of V3 interacting effectively with Cyc B, it is essential to mark its tripeptide Gly-15-Pro-16-Gly-17 occurring actually in all of the deciphered amino acid sequences of the HIV-1 principal neutralizing determinant [47]. Functional role of this invariant V3 stretch has not been completely specified. Nevertheless, it as known that, even a single substitution for its central residue by alanine makes an impact both on the virus immunogenicity and infectivity [48] testifying to important role of Pro-16 in the HIV-1 life cycle. Under the data derived, the 3D structure of the V3 fragment of interest is practically identical to that of Cyc B site Gly-1-Pro-2-Lys-3 which is spatially close to it: the value of cRMSD computed for all of the atoms of their main chains totals $0.7 \AA$. Resem- blance of the 3D main chain shapes observed for the two segments of the ligand and the receptor makes it possible to suggest that Cyc B stretch Gly-1-Pro-2-Lys-3 gives rise to the signal structure that is interpreted by V3 as a mirror image of its own immunogenic crest, which, most likely, presents the head reason involving the specificity of V3 interactions with immunophilins. In this light, the findings above confirm the validity of the assumption made in our previous studies [30,31] where specific high-affinity interactions of the HIV-1 V3 variable loops with immunophilins arising from experimental observa- tions [13] were suggested to be stipulated by appearing in their amino acid sequences the fragments exposing the similar 3D structures which are constructed from $\beta$-turns of polypeptide chain (for details see works [30,31]).

In such a way, the data of molecular docking testify to realizing the energetically favorable contacts of the HIV-1 SA-V3 loop with Cyc B resulting in the masking of some of the key V3 amino acids of its immunogenic crown. In this context, we could suggest of a possible usage of immunophilins and, in particular, $\mathrm{Cyc} \mathrm{B}$ as an alternative to the V3-directed antibodies commonly used to neutralize the HIV-1 activity. However, the evidence of study [49] demonstrating that increase of immunophilins concentration in infected blood plasma does not influence the virus infectivity conflicts with this primitive conjecture. In the case of Cyc B, the probable cause of its insufficient neutralizing activity may consist in the fact that, as follows from our simulations, the binding of the immunophilin to the HIV-1 V3 loop occurs via interactions with the central region of $\mathrm{V} 3$ and does not affect its N- and C-terminals (Figure 1) where the major portion of the residues involved in cell tropism and cell fusion is localized [50-52]. Therefore, to amplify the blockade of V3 and preserve its capacity for specific interactions with Cyc B, we have undertaken an attempt to design as potential anti-HIV-1 drug the virtual molecule named Cyc B peptide and imitating N-terminal segment 1-30 of the native immunophilin. The choice of Cyc B segment 1-30 for continuation of our studies is caused by the following motive: in compliance with the designed data, it holds tripeptide Gly-1-Pro-2-Lys-3 recognizable by the virus immunogenic crest and comprises significant share of the residues making the binding site for V3. Certainly, such a definition is correct only in case that the 3D structure of this Cyc B segment does not experience the considerable alterations in its free state. To check whether that is true, we computed the most preferable 3D structure of the Cyc B peptide and compared it with the one appearing in crystal [37] in the corresponding site of the intact protein. Analysis of Figure 2 illustrating the image of the superposed peptide structures gives grounds to conclude that the spatial folds of their backbone are closely related, and this inference arising from visual observation is ratified by the value of cRMSD equal to $2.4 \AA$. At collating the structures given in Figure 2, it is essential to underscore that very close agreement between them (cRMSD is $0.5 \AA$ ) occurs in segment Gly-1-Pro-2-Lys-3 that, as stated above, forms in the native $\mathrm{Cyc} \mathrm{B}$ the conformational epitope specifically recognizable by V3. Analogous conclusion to the effect that the compared structures are alike follows from their confrontation in the conformational space $(\phi, \psi)$ : the value of aRMSD calculated for all of the peptide amino acids comes to $33^{\circ}$.

Insight into the static model for the 3D structure of the Cyc B peptide (Figure 3(a)) shows that essential contribution to its energy stabilization belongs to the donoracceptor interactions that result in forming the extensive network of hydrogen bonds appearing between amino acids both distant and adjacent in the polypeptide chain. The molecule generated by computer modeling tools offers the elongated "construction" in which the spatially close $\mathrm{N}$ - and $\mathrm{C}$-terminal residues give rise to the longrange $\mathrm{H}$-bond by the oxygen of Gly- 1 carboxyl group and the hydrogen of hydroxyl group of Thr-30 side chain. As follows from the dihedral values given in Table 2, central part 10-22 of the Cyc B peptide constitutes the $\beta$-sheet the "oval isthmus" of which is composed from consecutive $\beta$-turns (Figure 3(b)), with their spatial folds being similar to those previously [32] in the immunogenic crown of the HIV-1 SA-V3 loop. In particular, according to our simulations, tetrapeptide Ile-14-Gly-15Asp-16-Glu-17 of the Cyc B peptide adopts the conformation of none-standard $\beta$-turn IV close to that of stretch Gly-15-Pro-16-Gly-17-Gln-18 of V3: in this case, the 
Table 2. Dihedral angles for amino acids in the 3D structure of the Cyc B peptide.

\begin{tabular}{|c|c|c|c|c|c|}
\hline \multirow{2}{*}{ Residue } & \multicolumn{5}{|c|}{ Dihedral angles (deg.) } \\
\hline & $\phi$ & $\psi$ & $\chi_{1}$ & $\chi_{2}$ & $\chi_{3}$ \\
\hline Gly-1 & - & -109.7 & - & - & - \\
\hline Pro-2 & -52.2 & 178.6 & -18.1 & 31.0 & \\
\hline Lys-3 & -135.3 & 170.1 & -64.6 & -176.2 & -69.6 \\
\hline Val-4 & -84.6 & 141.8 & 176.6 & - & - \\
\hline Thr-5 & -135.9 & -136.8 & 69.1 & - & - \\
\hline Val-6 & -60.6 & 123.4 & -62.0 & - & - \\
\hline Lys-7 & -93.7 & 80.2 & -173.1 & 59.0 & 168.0 \\
\hline Val-8 & -112.5 & 160.8 & -153.8 & - & - \\
\hline Tyr-9 & -78.7 & 170.2 & 78.2 & -82.8 & - \\
\hline Phe-10 & -144.3 & 161.7 & -168.0 & -101.0 & - \\
\hline Asp-11 & -85.7 & 165.1 & -141.6 & -58.4 & - \\
\hline Leu-12 & -147.4 & 101.0 & -154.5 & -50.8 & - \\
\hline Arg-13 & -129.4 & 102.0 & -45.8 & -162.4 & 70.9 \\
\hline Ile-14 & -80.3 & 88.7 & -55.0 & -179.9 & - \\
\hline Gly-15 & 74.1 & -72.1 & - & - & - \\
\hline Asp-16 & -147.8 & 5.8 & -152.3 & -61.9 & - \\
\hline Glu-17 & -53.7 & 120.6 & -64.8 & -176.4 & -30.7 \\
\hline Asp-18 & -74.2 & 42.0 & -157.7 & -137.1 & - \\
\hline Val-19 & -52.9 & -31.1 & -165.5 & - & - \\
\hline Gly-20 & 118.2 & -175.8 & - & - & - \\
\hline Arg-21 & -80.9 & 55.8 & -75.7 & 157.4 & -70.7 \\
\hline Val-22 & -53.2 & 129.0 & 179.8 & - & - \\
\hline Ile-23 & -76.6 & 60.7 & -39.7 & -59.3 & - \\
\hline Phe-24 & -56.9 & -63.3 & -66.1 & -75.9 & - \\
\hline Gly-25 & 77.9 & 171.0 & - & - & - \\
\hline Leu-26 & -138.0 & 159.7 & -66.5 & 95.1 & - \\
\hline Phe-27 & -97.0 & -5.3 & -51.8 & -83.5 & - \\
\hline Gly-28 & 61.7 & -130.8 & - & - & - \\
\hline Lys-29 & -133.8 & 143.2 & 176.9 & 63.7 & 169.4 \\
\hline Thr-30 & -122.6 & - & -62.2 & - & - \\
\hline
\end{tabular}

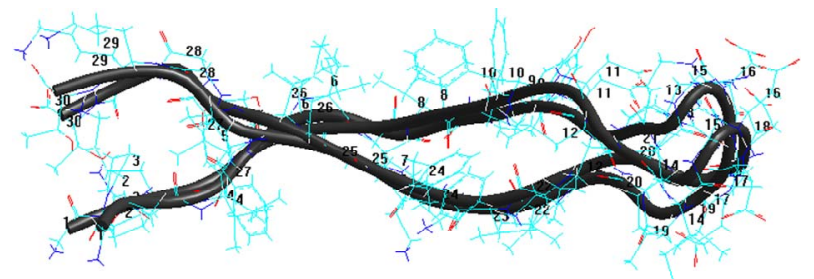

Figure 2. 3D structure of the Cyc B peptide superposed with the X-ray conformation for segment 1-30 of the entire protein.

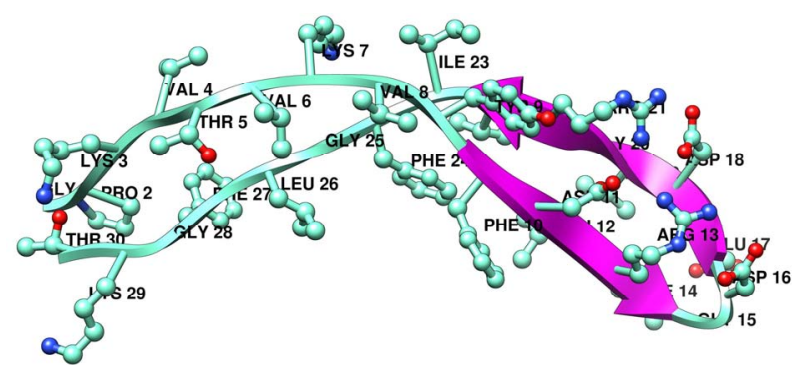

(a)

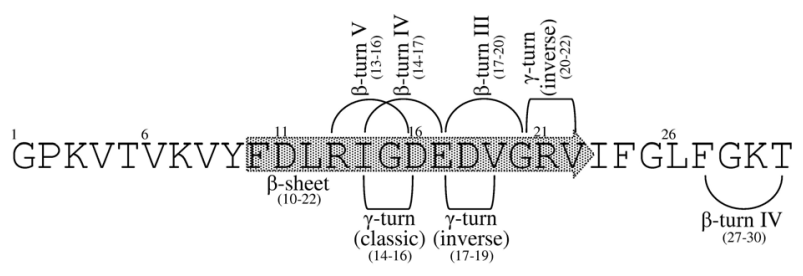

(b)

Figure 3. (a) Three-dimensional; (b) structures of the Cyc B peptide generated based on the X-ray conformation of site 1-30 for the intact Cyc B.

value of cRMSD estimated for the backbone atoms of the compared structures is $1.1 \AA$. Resemblance in the structural organization of the central regions of $\mathrm{V} 3$ and the Cyc B peptide takes also place for their longer fragments. For instance, if we compare the 3D structure of V3 stretch 15-20 producing the overwhelming majority of contacts with neutralizing antibodies (study [53]) with the one of the Cyc B peptide segment 14-19, it is also possible to observe the conformity of their spa- tial backbone folds (the corresponding value of cRMSD aggregates $2.0 \AA$ ). This outcome enables one to assume that, subject to the observance of the principle of "mirror similarity" formulated in studies [30,31], segment of the Cyc B peptide forming the "oval isthmus" of the $\beta$-sheet and located in the native protein inside its globule may give an additional site for specific binding to V3 alternative to stretch Gly-1-Pro-2-Lys-3 of the intact immunophilin.

When looking into the secondary structure of the designed molecule (Figure 3(b)), one cannot but catch sight of the peptide segment 27-30 that, as the stretches 
of its central part, exposes the conformation of $\beta$-turn, which merits the principal concern in view of the data on the 3D structure of the SA-V3 loop [32] where the C-terminal site organizes exactly the same structural motif. This evidence combined with that above implicates the following conclusion: the secondary structures of V3 and the Cyc B peptide observed in their central and C-terminal portions are closely related. In addition, considering the main chain dihedrals (Table 2) indicates that, except $\beta$-turns, the analyzed structure also forms $\gamma$-bends the central residues of which are located in positions 15,18 , and 21 (Figure 3(b)).

The results of molecular dynamics simulations implemented during $20 \mathrm{~ns}$ time domain by applying the static 3D structure of the Cyc B peptide as the starting model are evidence of its relative conformational rigidity: the average of cRMSD calculated for the structures of the MD trajectory and starting point amounts $3.1 \AA$ and the system of intramolecular H-bonds serving as one of the factors stabilizing the molecule structure undergoes no drastic changes within the whole MD trajectory. Nonetheless, comprehensive study on the dynamic structures of Cyc B peptide indicates that their individual representatives differ significantly from the input structure, which does not exclude the probability of wide-ranging structural reorganizations of this molecule stimulated by abrupt alterations of the environment that, for example, may happen upon its entry into numerous intermolecular interactions.

Conformity of the 3D structure of the Cyc B peptide with that of the same immunophilin segment (Figure 2) and its relative structural inflexibility following from the data of molecular dynamics computations give ground to believe that the molecule designed here may not only conserve the capacity for specific interaction with V3 characteristic of the native protein [13] but also intensify the blockade of this cryptic site of gp120. Indeed, delving into the potential energy function describing the structural complex of the Cyc B peptide with V3 illustrates (Figure 4) that, as compared to the native protein, the peptide originating from its framework exhibits much more extensive network of contacts with the V3 segments embracing the biologically significant amino acids of gp120. So the energy of intermolecular interacttions in the complex of Cyc B with V3 amounts to -75 $\mathrm{kcal} / \mathrm{mol}$ and, in the case of interest, its value falls down to $-350 \mathrm{kcal} / \mathrm{mol}$. And at the same time, stabilization of the complex between the Cyc B peptide and V3 is reached owing to the multiple donor-acceptor interactions (see Table 3) as well as to the salt bridge formed using Arg13 of V3 and Asp-18 of immunophilin-derived peptide. When analyzing the system of $\mathrm{H}$-bonds given in Table 3, there is need to note that, from the side of V3, contribution to its formation belongs to such biologically meaningful residues of gp120 as Lys-10, Arg-13, Gly-17, Gln-18, Asp-25, Asp-29, Ile-30, and Arg-31 which find themselves to be isolated as a result of arising the overmolecular ensemble. Among the residues of this register, we ought to notice Asp-25 that takes an active part in binding of the virus to the cell membrane surface [54-58] and, along with Ser-11, accounts for the HIV-1 phenotype $[59,60]$. Constituting the complex of the Cyc B peptide with V3 also entails the masking of its functionally critical amino acids Ser-11, Ala-19, Ile-23, Gly-24, and Gln-32 which are also utilized by the virus to set up the cell tropism determinant [54-58]. The active center of $\mathrm{V} 3$ responsible for binding to $\mathrm{Cyc} \mathrm{B}$ peptide contains Asn-6 the blockade of which may be highly effective for the virus inactivation: as mentioned above, this amino acid of V3 presenting the integral part of its structurally invariant segment 3-7 [32] gives rise to one of the conserved sites of N-linked glycosylation of gp120 [61].

When examining the overmolecular ensemble represented in Figure 4, one needs to cast a glance at the following feature: in this complex, segment Gly-1-Pro-2Lys-3 of the Cyc B peptide interacts with structurally rigid stretch 28-32 of the HIV-1 V3 domain [32], whereas the corresponding site of the native protein, as stated before, contacts the immunogenic crown of gp120 (Figure 1). At the same time, this $\mathrm{V} 3$ region that proves to be unused by the N-terminal site of the Cyc B peptide becomes very intimate with pentapeptide Ile-14-Gly-15Asp-16-Glu-17-Asp-18 belonging to the "oval isthmus" of its $\beta$-sheet. These observations are of special interest since the indicated segments of the receptor and ligand reside in the $\beta$-turns of polypeptide chain which may serve as docking sites for protein-protein interactions [62-64]. The presence of $\beta$-turns in the 3D structures of V3 and the Cyc B peptide is likely to be one of the head factors that may make a determinative contribution to the specificity of their efficacious interactions.

Collating the 3D structures of V3 and the Cyc B peptide in natural and constrained states indicates that, in either case, forming the complex brings in the certain structural rearrangements taking place both in the Cartesian and angular spaces. At the same time, the Cyc B peptide experiences the more profound transformation of its structure: so when we confront the V3 structures materialized in the overmolecular ensemble and in the unbound status, the values of cRMSD and aRMSD are respectively $2.0 \AA$ and $47^{\circ}$ and, in the case of the Cyc B peptide, the corresponding values rise to $4.0 \AA$ and $57^{\circ}$. This observation falls into line with the supposition above, whereby the Cyc B peptide, in spite of the relative conformational rigidity, may exhibit the higher flexibility of the polypeptide chain on drastic medium alterations. 


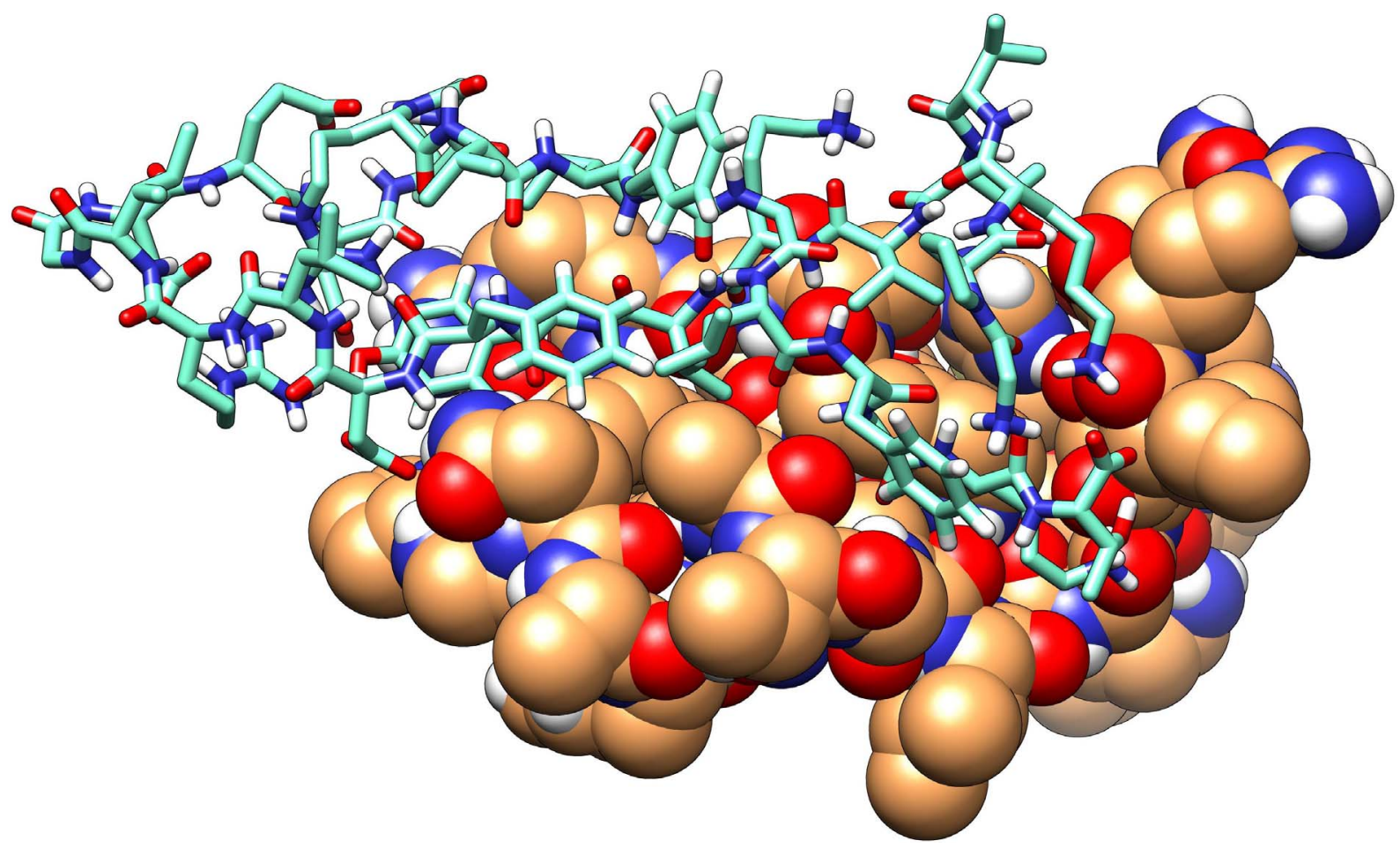

Figure 4. Overmolecular structure of the HIV-1 SA-V3 loop (balls) with the Cyc B peptide (tubes).

Table 3. Geometric parameters of intermolecular H-bonds for the structural complex of the HIV-1 SA-V3 loop with the Cyc B peptide.

\begin{tabular}{|c|c|c|c|c|c|}
\hline $\begin{array}{l}\text { Residue } \\
\text { (donor) }\end{array}$ & $\begin{array}{l}\text { Group } \\
\text { (donor) }\end{array}$ & $\begin{array}{l}\text { Residue } \\
\text { (acceptor) }\end{array}$ & $\begin{array}{l}\text { Group } \\
\text { (acceptor) }\end{array}$ & $\begin{array}{c}\text { Distance }(\AA) \\
\text { Donor...Acceptor }\end{array}$ & $\begin{array}{c}\text { Distance }(\AA) \\
\text { Hydrogen...Acceptor }\end{array}$ \\
\hline Arg- $13^{1}$ & NH1 & Asp- $18^{2}$ & OD1 & 3.0 & 2.0 \\
\hline Arg- $13^{1}$ & $\mathrm{NH} 2$ & Asp- $18^{2}$ & OD1 & 3.2 & 2.3 \\
\hline Arg-13 ${ }^{1}$ & $\mathrm{NH} 2$ & Asp- $18^{2}$ & OD2 & 3.1 & 2.2 \\
\hline Gly- $17^{1}$ & $\mathrm{NH}$ & Asp $11^{2}$ & OD1 & 2.9 & 1.9 \\
\hline Gln- $18^{1}$ & NE2 & Tyr- $-9^{2}$ & $\mathrm{CO}$ & 2.8 & 1.8 \\
\hline Gln- $18^{1}$ & NE2 & Asp- $11^{2}$ & OD1 & 2.8 & 1.8 \\
\hline Asp- $25^{1}$ & $\mathrm{NH}$ & Gly-28 ${ }^{2}$ & $\mathrm{CO}$ & 2.8 & 1.8 \\
\hline Lys- $3^{2}$ & $\mathrm{NZ}$ & Ile- $30^{1}$ & $\mathrm{CO}$ & 2.8 & 1.9 \\
\hline Thr- $5^{2}$ & OG1 & Arg- $31^{1}$ & $\mathrm{CO}$ & 2.7 & 1.7 \\
\hline Lys- $7^{2}$ & $\mathrm{NH}$ & Lys- $10^{1}$ & $\mathrm{CO}$ & 2.8 & 1.8 \\
\hline Lys- $29^{2}$ & $\mathrm{NZ}$ & Asp- $29^{1}$ & $\mathrm{CO}$ & 2.9 & 2.0 \\
\hline
\end{tabular}

Footnote: Superscripts 1 and 2 denote the amino acids of $\mathrm{V} 3$ and the CycB peptide respectively.

\section{CONCLUSIONS}

In studies [30,31], we implemented the computer-aided design of two molecules referred to as Cyc A and FKBP peptides and, having analyzed their structural complexes with the HIV-1 SA-V3 loop [32], disclosed that the Cyc
A peptide binds effectively to its immunogenic crown, whereas the FKBP peptide prefers to interact with the Nand $\mathrm{C}$-terminal segments of the virus principal neutralizing determinant. The findings derived here bear witness that, unlike the molecules constructed previously [30,31], the Cyc B peptide is able to mask the function- 
ally crucial amino acids both of the V3 central part and of its stem stretches. Moreover, as compared to these molecules, cooperation of the Cyc B peptide with V3 brings in the origin of more stable overmolecular structure: for instance, the value of the energy of intermolecular interactions computed for the structural complex of V3 with Cyc A peptide [31] totals $-87 \mathrm{kcal} / \mathrm{mol}$ and, in the case in question, it aggregates $-350 \mathrm{kcal} / \mathrm{mol}$ (see above).

As shown in study [32], in spite of the hypervariability of V3, its segments 3-7, 15-20, and 28-32 embracing the highly conserved amino acids of gp120 give rise to the closely related spatial backbone folds in different virus isolates, and, therefore, they may be considered as promising targets for anti-AIDS drug studies. Allowing for these data in common with the evidence which bears witness that the Cyc B peptide is capable of masking the V3 functionally critical residues residing in its structurally invariant segments, one may expect that synthetic copy of this virtual molecule (or its structural analogs) may display biological activity to various HIV-1 strains exhibiting a broadly neutralizing effect. Beyond all shadow of doubt, the peptide constructed here must experience the extensive experimental test to be considered as the coming applicant for the role of "magic bullet" displaying a wide-range blockade of the HIV-1 envelope glycoprotein gp120.

In conclusion, the model of the structural complex of Cyc B with V3 proposed above substantiates the literature data on a high affinity of immunophilins to V3 [13], and the results derived from its analysis enable one to make an optimistic prognosis of the prospects of using their peptides as the starting chemicals for the design of efficacious antiviral agents by protein engineering methods.

\section{ACKNOWLEDGEMENTS}

This study was supported by grants from the Union State of Russia and Belarus (scientific program SKIF-GRID; № 4U-S/07-111) as well as from the Belarusian Foundation for Fundamental Research (project X08-003).

\section{REFERENCES}

[1] Gallo, R.C. and Montagnier, L. (2003) The discovery of HIV as the cause of AIDS. The New England Journal of Medicine, 349(24), 2283-2285.

[2] Wyatt, R. and Sodroski, J. (1998) The HIV-1 envelope glycoproteins: Fusogens, antigens, and immunogens. Science, 280(5371), 1884-1888.

[3] Landau, N.R., Warton, M. and Littman, D.R. (1988) The envelope glycoprotein of the human immunodeficiency virus binds to the immunoglobulin-like domain of CD4 .
Nature, 334(6178), 159-162.

[4] Feng, Y., Broder, C.C., Kennedy, P.E. and Berger, E.A. (1996) HIV-1 entry cofactor: Functional cDNA cloning of a seven-transmembrane, $\mathrm{G}$ protein-coupled receptor. Science, 272(5263), 872-877.

[5] Deng, H., Liu, R., Ellmeier, W., Choe, S., Unutmaz, D., Burkhart, M., Di Marzio, P., Marmon, S., Sutton, R.E., Hill, C.M., Davis, C.B., Peiper, S.C., Schall, T.J., Littman, D.R. and Landau, N.R. (1996) Identification of a Major Co-Receptor for Primary Isolates of HIV-1. Nature, 381(6584), 661-666.

[6] Sirois, S., Sing, T. and Chou, K.C. (2005) HIV-1 gp120 V3 loop for structure-based drug design. Current Protein \& Peptide Science, 6(5), 413-422.

[7] Hartley, O., Klasse, P.J., Sattentau, Q.J. and Moore J.P. (2005) V3: HIVs Switch-Hitter. AIDS Research and Human Retroviruses, 21(8), 171-189.

[8] Hwang, S.S., Boyle, T.J., Lyerly, H.K. and Cullen, B.R. (1991) Identification of the envelope V3 loop as the primary determinant of cell tropism in HIV-1. Science, 253(5015), 71-74.

[9] Choe, H., Farzan, M., Sun, Y., Sullivan, N., Rollins, B., Ponath, P.D., Wu, L., Mackay, C.R., LaRosa, G., Newman, W., Gerard, N., Gerard, C. and Sodroski, J. (1996) The beta-chemokine receptors CCR3 and CCR5 facilitate infection by primary HIV-1 isolates. Cell, 85(7), 11351148.

[10] Cocchi, F., DelVico, A., Garzino-Demo, A., Cara, A., Gallo, R.C. and Lusso, P. (1996) The V3 domain of the HIV-1 gp120 envelope glycoprotein is critical for chemokine-mediated blockade of infection. Nature Medicine, 2(11), 1244-1247.

[11] Connor, R.I., Sheridan, K.E., Ceradini, D., Choe, S. and Landau, N.R. (1997) Change in coreceptor use correlates with disease progression in HIV-1-infected individuals. The Journal of Experimental Medicine, 185(4), 621-628.

[12] Scarlatti, G., Tresoldi, E., Bjorndal, A., Fredriksson, R., Colognesi, C., Deng, H.K., Malnati, M.S., Plebani, A., Siccardi, A.G., Littman, D.R., Fenyo, E.M. and Lusso, P. (1997) In vivo evolution of HIV-1 co-receptor usage and sensitivity to chemokine-mediated suppression. Nature Medicine, 3(11), 1259-1265.

[13] Endrich, M.M. and Gehring, H. (1998) The V3 loop of human immunodeficiency virus type-1 envelope protein is a high-affinity ligand for immunophilins present in human blood. European Journal of Biochemistry, 252(3), 441-446.

[14] Galat, A. and Metcalfe, S.M. (1995) Peptidylproline cis/trans isomerases. Progress in Biophysics and Molecular Biology, 63(1), 67-118.

[15] Barik, S. (2006) Immunophilins: For the love of proteins. Cellular and Molecular Life Sciences, 63(24), 28892900.

[16] Baker, E.K., Colley, N.J. and Zuker, C.S. (1994) The cyclophilin homolog NinaA functions as a chaperone, forming a stable complex in vivo with its protein target rhodopsin. The EMBO Journal, 13(20), 4886-4895.

[17] Ferreira, P.A., Nakayama, T.A. and Travis, G.H. (1997) Interconversion of red opsin isoforms by the cyclophilinrelated chaperone protein Ran-binding protein 2. Proceedings of the National Academy of Sciences, USA, 94(4), 1556-1561. 
[18] Hacker, J. and Fischer, J. (1993) Immunophilins: structure-function relationship and possible role in microbial pathogenicity. Molecular Microbiology, 10(3), 445-456.

[19] Moro, A., Ruiz-Cabello, F., Fernandez-Cano, A., Stock, R. P. and Gonzalez, A. (1995) Secretion by Trypanosoma cruzi of a peptidyl-prolyl cis-trans isomerase involved in cell infection. The EMBO Journal, 14(11), 2483-2490.

[20] Spik, G., Haendler, B., Delmas, O., Mariller, C., Chamoux, M., Maes, P., Tartar, A., Montreuil, J., Stedman, K., Kocher, H.P., Keller, R., Hiestand, P.C. and Movva, N.R. (1991) A novel secreted cyclophilin-like protein (SCYLP). Journal of Biological Chemistry, 266(17), 10735-10738.

[21] Allain, F., Boutillon, C., Mariller, C. and Spik, G. (1995) Selective assay for CyPA and CyPB in human blood using highly specific anti-peptide antibodies. Journal of Immunological Methods, 178(1), 113-120.

[22] Endrich, M. M., Grossenbacher, D., Geistlich, A. and Gehring, H. (1996) Apoptosis-induced concomitant release of cytosolic proteins and factors which prevent cell death. Biology of the Cell, 88(1-2), 15-22.

[23] Sherry, B., Yarlett, N., Strupp, A. and Cerami, A. (1992) Identification of cyclophilin as a proinflammatory secretory product of lipopolysaccharide-activated macrophages. Proceedings of the National Academy of Sciences, USA, 89(8), 3511-3515.

[24] Xu, Q., Leiva, M.C., Fischkoff, S.A., Handschumacher, R.E. and Lyttle, C.R. (1992) Leukocyte chemotactic activeity of cyclophilin. The Journal of Biological Chemistry, 267(17), 11968-11971.

[25] Bang, M., Muller, W., Hans, M., Brune, K., Swandulla, D. (1995) Activation of $\mathrm{Ca} 2+$ signaling in neutrophils by the mast cell-released immunophilin FKBP12. Proceedings of the National Academy of Sciences, USA, 92(8), 3435-3438.

[26] Saphire, A.C.S., Bobardt, M.D. and Gallay, P.A. (1999) Host cyclophilin A mediates HIV-1 attachment to target via heparans. The EMBO Journal, 18(23), 6771-6785.

[27] Franke, E.K., Yuan, H.E.H. and Luban, J. (1994) Specific incorporation of cyclophhilin a into HIV-1 virions. Nature, 372(6504), 359-362.

[28] Thali, M., Bukovsky, A., Kondo, E., Rosenwirth, B., Walsh, C.T., Sodroski, J. and Gottlinger, H.G. (1994) Functional association of cyclophilin A with HIV-1 virions. Nature, 372(6504), 363-365.

[29] Colgan, J., Yuan, H.E.H., Franke, E.K. and Luban, J. (1996) Binding of the human immunodeficiency virus type 1 Gag polyprotein to cyclophilin A is mediated by the central region of capsid and requires Gag dimerization. The Journal of Virology, 70(7), 4299-4310.

[30] Andrianov, A.M. (2008) Computational anti-AIDS drug design based on the analysis of the specific interactions between immunophilins and the HIV-1 gp120 V3 loop. Application to the FK506-binding protein. Journal of Biomolecular Structure \& Dynamics, 26(1), 49-56.

[31] Andrianov, A.M. (2009) Immunophilins and HIV-1 V3 loop for structure-based anti-AIDS drug design. Journal of Biomolecular Structure \& Dynamics, 26(4), 445-454.

[32] Andrianov A.M. and Anishchenko I.V. (2009) Computational model of the HIV-1 subtype A V3 loop: Study on the conformational mobility for structure-based antiAIDS drug design. Journal of Biomolecular Structure \& Dynamics, 27(2), 179-194.
[33] Bernstein, F.C., Koetzle, T.F., Williams, G.J.B., Meyer, E. F., Brice, M.D., Rodgers, J.R., Kennard, O., Shimanouchi, T. and Tasumi, M. (1997) The protein data bank. A computer-based archival file for macromolecular structures. Journal of Molecular Biology, 112(3), 535-542.

[34] Berman, H.M., Westbrook, J., Feng, Z., Gilliland, G., Bhat, T.N., Weissig, H., Shindyalov, I.N. and Bourne, P.E. (2000) The Protein Data Bank. Nucleic Acids Research, 28(1), 235-242.

[35] Mustard, D. and Ritchie, D.W. (2005) Macromolecular docking using spherical polar fourier correlations. Proteins: Structure, Function, and Bioinformatics, 60(2), 269-274.

[36] Berendsen, H.J.C., van der Spoel, D. and van Drunen, R. (1995) GROMACS: A message-passing parallel molecular dynamics implementation. Computer Physics Communications, 91(1-3), 43-56.

[37] Mikol, V., Kallen, J. and Walkinshaw, M.D. (1994) X-ray structure of a Cyclophilin B/Cyclosporin complex: Comparison with Cyclophilin A and delineation of its calcineurin-binding domain. Proceedings of the National Academy of Sciences, 91(11), 5183-5186.

[38] Ren, P. and Ponder, J.W. (2003) TINKER: Software tools for molecular design. The Journal of Biological Chemistry, 107, 5933-5947.

[39] Oostenbrink, C., Villa, A., Mark, A.E. and van Gunsteren, W.F. (2004) A biomolecular force field based on the free enthalpy of hydration and solvation: The GROMOS force-field parameter sets 53A5 and 53A6. The Journal of Biological Chemistry, 25(13), 1656-1676.

[40] Berendsen, H.J.C., Postma, J.P.M., van Gunsteren, W.F. and Hermans, J. (1981) Interaction models for water in relation to protein hydration. In: B. Pullman Ed., Intermolecular Forces, Dordrecht: D. Reidel Publishing Company, Boston, pp. 331-342.

[41] Berendsen, H.J.C., Postma, J.P.M., DiNola, A. and Haak, J.R. (1984) Molecular-dynamics with coupling to an external bath. Journal of Chemical Physics, 81(8), 36843690 .

[42] Ablameyko, S.V., Abramov, S.M., Anishchanka, U.V., Medvedev, S.V., Paramonov, N.N. and Tchij, O.P. (2005) SKIF supercomputer configurations (in Russian). Minsk, United Institute of Informatics Problems.

[43] Smith, L.J., Bolin, K.A., Schwalbe, H., MacArthur, M. W., Thornton, J.M. and Dobson, C.M. (1996) Analysis of main chain torsion angles in proteins: Prediction of NMR coupling constants for native and random coil conformations. Journal of Molecular Biology, 255(3), 494-506.

[44] Hutchinson, E.G. and Thornton, J.M. (1996) PROMOTIF - a program to identify and analyze structural motifs in proteins. Protein Science, 5(2), 212-220.

[45] Sherman, S.A. and Johnson, M.E. (1993) Derivation of locally accurate spatial protein structure from NMR data. Progress in Biophysics and Molecular Biology, 59(3), 285- 339.

[46] Cormier, E.G. and Dragic, T. (2002) The crown and the stem V3 loop play distinct roles in human immunodeficiency virus type 1 envelope glycoprotein interactions with CCR5 coreceptor. The Journal of Virology, 76(17), 8953-8957.

[47] LaRosa, G.J., Davide, J.P., Weinhold, K., Waterbury, J.A., Profy, A.T., Lewis, J.A., Langlois, A.J., Dressman, G.R., 
Boswell, R.N., Shadduk, P., Holley, L.H., Karplus, M., Bolognesi, D.P., Matthews, T.J., Emini, E.A. and Putney, S.D. (1990) Conserved sequence and structural elements in the HIV-1 principal neutralizing determinant. Science, 249(4971), 932-935.

[48] Ivanoff, L.A., Looney, D.J., McDanal, C., Morris, J.F., Wong-Staat, F., Lang, A.J., Petteway, S.R.Jr. and Matthews, T.J. (1991) Alteration of HIV-1 infectivity and neutralization by a single amino acid replacement in the V3 loop domain. AIDS Research and Human Retroviruses, 7(7), 595-603.

[49] Minder, D., Boni, J., Schupbach, J. and Gering, H. (2002) Immunophilins and HIV-1 infection. Archives of Virology, 147(8), 1531-1542.

[50] Wang, W.-K., Dudek, T., Zhao, Y.-J., Brumblay, H.G., Essex, M. and Lee, T.-H. (1998) CCR5 coreceptor utilization involves a highly conserved arginine residue of HIV type 1 gp120. Proceedings of the National Academy of Sciences, USA, 95(10), 5740-5745.

[51] de Parseval, A., Bobardt, M.D., Chatterji, U., Elder, J.H., David, G., Zolla-Pazner, S., Farzan, M., Lee, T.H. and Gallay, P.A. (2005) A highly conserved arginine in gp120 governs HIV-1 binding to both syndecans and CCR5 via sulfated motifs. The Journal of Biological Chemistry, 280(47), 39493-39504.

[52] Hu, Q., Napier, K.B., Trent, J.O., Wang, Z., Taylor, S., Griffin, G.E., Peiper, S.C. and Shattock, R.J. (2005) Restricted variable residues in the C-terminal segment of HIV-1 V3 loop regulate the molecular anatomy of CCR5 utilization. Journal of Molecular Biology, 350(4), 699. 712.

[53] Ghiara, J.B., Stura, E.A., Stanfield, R.L., Profy, A.T. and Wilson, I.A. (1994) Crystal structure of the principal neutralization site of HIV-1. Science, 264(5155), 82-85.

[54] Chavda, S.C., Griffin, P., Han-Liu, Z., Keys, B., Vekony, M.A. and Cann, A.J. (1994) Molecular determinants of the V3 loop of human immunodeficiency virus type 1 glyco-protein gp120 responsible for controlling cell tropism. Journal of General Virology, 75(11), 3249-3253.

[55] Mammano, F., Salvatori, F., Ometto, L., Panozzo, M., Chieco-Bianchi, L. and De Rossi, A. (1995) Relationship between the V3 loop and the phenotypes of human immunodeficiency virus type 1 (HIV-1) isolates from chil- dren perinatally infected with HIV-1. The Journal of Virology, 69(1), 82-92.
[56] Milich, L., Margolin, B.H. and Swanstrom, R. (1993) v3 loop of the human immunodeficiency virus type 1 env protein: Interpreting sequence variability. The Journal of Virology, 67(9), 5623-5634.

[57] Shioda, T., Levy, J.A. and Cheng-Mayer, C. (1992) Small amino acid changes in the V3 hypervariable region of gp120 can affect the T-cell-line and macrophage tropism of human immunodeficiency virus type 1. Proceedings of the National Academy of Sciences, 89(20), 9434-9438.

[58] Wu, L., Gerard, N.P., Wyatt, R., Choe, H., Parolin, C., Ruffin, N., Borsetti, A., Cardoso, A.A., Desjardin, E., Newman, Gerard, W.C. and Sodroski, J. (1996) CD4-induced interaction of primary HIV-1 gp120 glycoproteins with the chemokine receptor CCR-5. Nature, 384(6605), 179-183.

[59] Fouchier, R.A.M., Groenink, M., Kootstra, N.A., Tersmette, M., Huisman, H.G., Miedema, F. and Schuitemaker, H. (1992) Phenotype-associated sequence variation in the third variable domain of the human immunodeficiency virus type 1 gp120 molecule. The Journal of Virology, 66(5), 3183-3187.

[60] De Jong, J.J., De Ronde, A., Keulen, W., Tersmette, M. and Goudsmit, J. (1992) Minimal requirements for the human immunodeficiency virus type $1 \mathrm{~V} 3$ domain to support the syncytium-inducing phenotype: Analysis by single amino acid substitution. The Journal of Virology, 66(11), 6777-6780.

[61] Ogert, R.A., Lee, M.K., Ross, W., Buckler-White, A., Martin, M.A. and Cho, M.W. (2001) N-linked glycosylation sites adjacent to and within the V1/V2 and the V3 loops of dualtropic human immunodeficiency virus type 1 isolate DH12 gp120 affect coreceptor usage and cellular tropism. The Journal of Virology, 75(13), 5998-6006.

[62] Smith, J.A. and Pease, L.J. (1980) Reverse turns in peptides and proteins. Critical Reviews in Biochemistry, 8(4), 315-399.

[63] Rose, G.D., Gierasch, L.M. and Smith, J.A. (1985) Turns in peptides and proteins. Advances in Protein Chemistry, 37, 1-109.

[64] Newton, A.C. (2001) Protein kinase C: Structural and spatial regulation by phosphorylation, cofactors, and macromolecular interactions. Chemical Reviews, 101(8), 2353-2364. 